\title{
Nonlinear Modeling of an Immersed Transmitting Capacitive Micromachined Ultrasonic Transducer for Harmonic Balance Analysis
}

\author{
H. Kagan Oguz, Selim Olcum, Student Member, IEEE, Muhammed N. Şenlik, Student Member, IEEE, \\ Vahdettin Taş, Abdullah Atalar, Fellow, IEEE, and Hayrettin Köymen, Senior Member, IEEE
}

\begin{abstract}
Finite element method (FEM) is used for transient dynamic analysis of capacitive micromachined ultrasonic transducers (CMUT) and is particularly useful when the membranes are driven in the nonlinear regime. One major disadvantage of FEM is the excessive time required for simulation. Harmonic balance (HB) analysis, on the other hand, provides an accurate estimate of the steady-state response of nonlinear circuits very quickly. It is common to use Mason's equivalent circuit to model the mechanical section of CMUT. However, it is not appropriate to terminate Mason's mechanical LC section by a rigid piston's radiation impedance, especially for an immersed CMUT. We studied the membrane behavior using a transient FEM analysis and found out that for a wide range of harmonics around the series resonance, the membrane displacement can be modeled as a clamped radiator. We considered the root mean square of the velocity distribution on the membrane surface as the circuit variable rather than the average velocity. With this definition, the kinetic energy of the membrane mass is the same as that in the model. We derived the force and current equations for a clamped radiator and implemented them using a commercial HB simulator. We observed much better agreement between FEM and the proposed equivalent model, compared with the conventional model.
\end{abstract}

\section{INTRODUCTION}

$\mathrm{C}$ AapaCITIVE micromachined ultrasonic transducers $\mathcal{U}$ (CMUTs) [1] were widely studied and fabricated in the past decade. CMUTs with some unique capabilities attracted the attention of researchers working with applications such as medical imaging, high-intensity focused ultrasound, intravascular ultrasound, airborne acoustics, microphones, and nondestructive evaluation. Fabrication of CMUTs for those applications requires tedious process steps, which are time consuming and expensive [2], [3]. Therefore, an accurate and fast simulation method is needed for designing CMUTs.

The efforts for simulating the CMUTs have started with the development of an equivalent circuit model [1],

Manuscript received April 10, 2009; accepted October 9, 2009. This work is supported in part by the Turkish Scientific and Research Council (TUBITAK) under project grants 105E23 and 107T921. SO acknowledges the support of TUBITAK and ASELSAN for their Ph.D. Scholarship Programs. AA thanks TUBA for the research support.

The authors are with the Electrical and Electronics Engineering Department, Bilkent University, Ankara, Turkey (e-mail: koymen@ ee.bilkent.edu.tr).

Digital Object Identifier 10.1109/TUFFC.2010.1424 based on Mason's equivalent circuit [4] for electroacoustic devices. Different models for defining the equivalent circuit elements are available in the literature [1], [5][7]. However, finite element method (FEM) simulations are still needed [8] to simulate the CMUT operation, including the nonlinear effects, medium loading, cross talk, and the effect of the higher order harmonics. Recently, fully analytical models are developed for fast and efficient results of frequency response analysis [9]. FEM simulation packages such as ANSYS (ANSYS Inc. Canonsburg, PA) are powerful tools and extensively used for the analysis of CMUTs. FEM analysis predicts the performance of a particular design accurately, and hence, it is a very good testing and tuning tool. However, the computational expense required for the solution makes FEM tools unsuitable for using them in design stage. For instance, transient dynamic analysis of a CMUT is crucial to understanding the nonlinear behavior of the CMUT. However, it has high computational cost and requires many cycles to reach the steady state. It does not rapidly respond when a parameter is altered, and hence, an idea about its effect cannot be instantly grasped by the designer. Calculated design charts for a large array of circular CMUTs are available in the literature [10] but lack the nonlinear effects when the CMUTs are driven with a high excitation voltage. In recent years, the efforts to model the nonlinear behavior of CMUTs under large excitations [6], [11] have increased due to the emerging need for applications that require nonlinear excitation [12]-[16].

In this work, we have developed an equivalent circuit, which takes into account the nonuniform velocity distribution across a membrane and predicts the nonlinear behavior of a circular CMUT. This model is based only on the physics of the device and does not employ FEM for any parameter determination. The equivalent circuit accurately includes the effect of the immersion medium loading. A linear equivalent circuit model can predict the small signal behavior of CMUT. However, CMUT exhibits strong nonlinear behavior even at very low AC excitation. A linear mechanical section in the equivalent circuit with a consistent radiation impedance is solved by harmonic balance (HB) analysis. We perform transient and harmonic balance simulations and show that the results are consistent with the FEM results. 


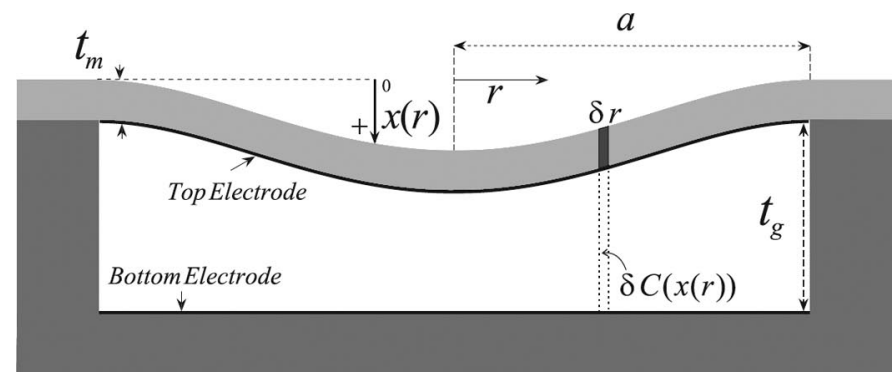

Fig. 1. 2-D view of the circular CMUT geometry. Top electrode is placed at the bottom of the membrane. The dimensional parameters of the CMUT and the cross-section of an infinitesimal ring on the membrane are depicted.

We have demonstrated the use of the proposed method by designing the time-waveform of the excitation signal to obtain the given acoustic radiation signal at the output of the CMUT.

\section{Analytical Equivalent Circuit Model}

It is common to use Mason's equivalent circuit to model the mechanical section of a CMUT [1], [4]. Mason's circuit is comprised of a series $L C$ section, where $L$ represents the equivalent mass, which is 1.8 times the membrane mass, and $C$ stands for the inverse of the spring constant of the membrane. In this equivalent circuit, the through and across variables are the average particle velocity and the total force on the driven surface of the membrane, respectively. In vacuum, where the medium loading is zero, Mason's mechanical section accurately models the CMUT and agrees with the FEM simulation results [5].

When the device is immersed, it is necessary to consider the terminating radiation impedance in the equivalent circuit to represent the device behavior correctly. The radiation impedance of a radiator is determined by the particle velocity distribution across its aperture. It is the ratio of total power radiated from the transducer to the square of the absolute value of a nonzero reference velocity. Therefore, the through and across lumped variables must be defined in such a way that they are consistent both in the equivalent circuit model and in the radiation impedance.

\section{A. Velocity Profile and the Radiation Impedance}

The acoustic radiation from radiators with nonuniform velocity profiles is studied by Greenspan [17]. The particle displacement and the velocity profile across circular clamped membranes are not uniform and can be very well approximated by the profiles that Greenspan studied:

$$
v(r)=(n+1) v_{\mathrm{avg}}\left[1-\frac{r^{2}}{a^{2}}\right]^{n} \text { for } r<a,
$$

where $a$ is the radius of the aperture, $r$ is the radial position, $v_{\text {avg }}$ is the average velocity along the membrane

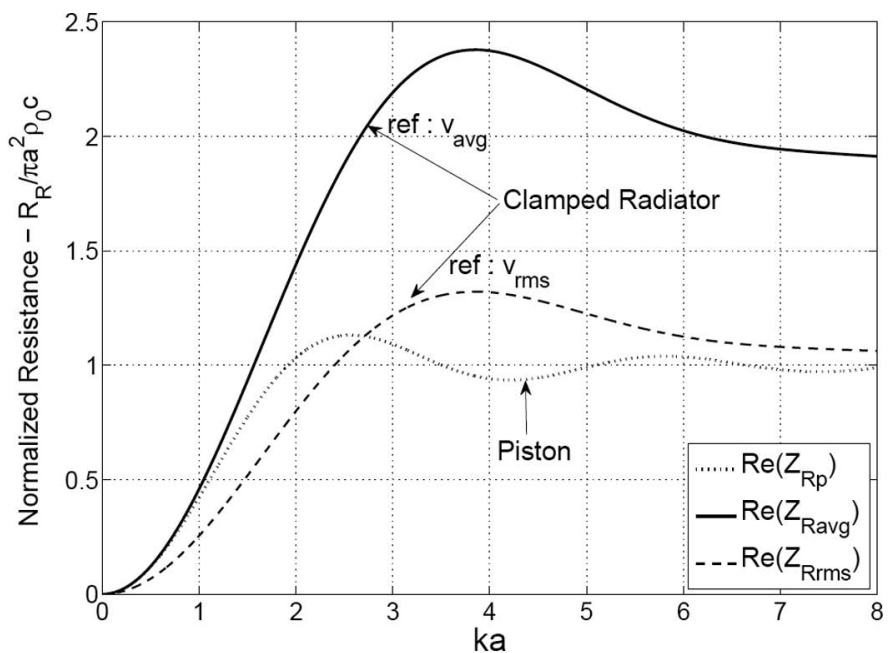

(a)

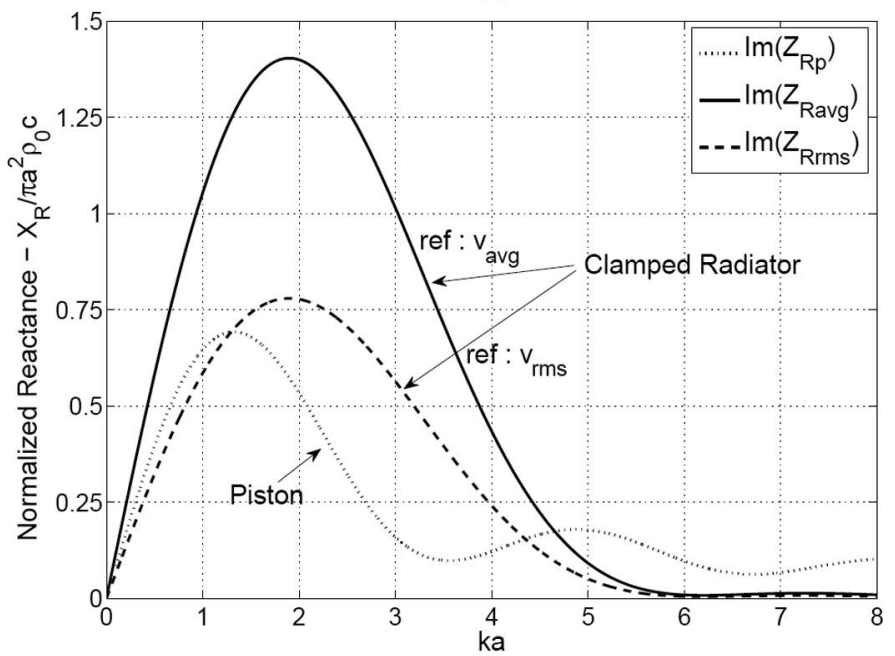

(b)

Fig. 2. (a) Real (resistive) and (b) imaginary (reactive) parts of the radiation impedance of the piston and the clamped radiator normalized to $\pi a^{2} \rho_{0} c$, where $\rho_{0}$ and $c$ are the density and the velocity of sound in the immersion medium. For a clamped radiator, both average and rms velocity of the membrane are used as the reference velocity and shown separately.

surface, and $n$ is a constant that specifies the type of the profile. If $n=0$, then (1) stands for the profile of a rigid piston. For a clamped membrane, as the CMUT shown in Fig. 1, $n=2$ approximates the nonuniform profile. The radiation impedance of a velocity profile similar to that of a CMUT is given as normalized radiated power of a clamped radiator $(n=2)$ in [17].

When the CMUT is immersed in water, it is reasonable to assume that the electro-mechanical behavior, hence the model, of the membrane remains intact. However, interaction between the membrane and the medium loads the mechanical section. In Fig. 2, the real and imaginary parts of the normalized radiation impedance of the piston radiator, $Z_{\mathrm{Rp}}$, and the clamped radiator, $Z_{\mathrm{Ravg}}$, are shown with respect to $k a$, where $k$ is the wave number. Mason's circuit and the radiation impedance $Z_{\text {Ravg }}$ are compatible, because the through lumped variable in both is the average 
velocity, $v_{\text {avg. }} Z_{\text {Ravg }}$ is considerably different than $Z_{\mathrm{Rp}}$ of a piston as depicted in Fig. 2. For example, the real part of $Z_{\text {Ravg }}$ is 1.8 times that of $Z_{\mathrm{Rp}}$ for large $k a$.

The inadequacy of simply terminating Mason's mechanical $L C$ section by a rigid piston's radiation impedance in water, to model the immersed CMUT, is demonstrated in [18], [19]. This is due to the fact that a rigid piston has a uniform velocity profile $(n=0)$, whereas CMUT can be better approximated by $n=2$ in (1). The real part (resistive) of $Z_{\mathrm{Rp}}$ and $Z_{\text {Ravg }}$ are similar for $k a<1$, as depicted in Fig. 2(a), whereas the reactive parts are not. In Mason's equivalent circuit, the turns ratio of the electromechanical transformer and the input capacitance are derived with parallel plate assumption, but the mechanical impedance is found based on the clamped membrane boundary conditions [4]. Considering all these factors, it is not appropriate to combine Mason's model with the piston radiation impedance.

\section{B. Root Mean Square Velocity}

The average velocity is not an appropriate lumped variable to determine the kinetic energy of the membrane, which is a distributed system. The kinetic energy calculated using the mass of the membrane and average velocity is less than the actual energy of the membrane. The kinetic energy, $E_{K}$, of the membrane mass is

$$
\begin{aligned}
E_{K} & =\int_{0}^{2 \pi} \int_{0}^{a} \frac{1}{2} v(r) v^{*}(r) \rho t_{m} r \mathrm{~d} r \mathrm{~d} \theta \\
& =\frac{1}{2}\left(\rho t_{m} \pi a^{2}\right)\left[\frac{1}{\pi a^{2}} \int_{0}^{2 \pi} \int_{0}^{a} v(r) v^{*}(r) r \mathrm{~d} r \mathrm{~d} \theta\right]
\end{aligned}
$$

where $v(r)$ is the velocity normal to the surface of the membrane, $\rho$ is the density, $t_{m}$ is the thickness, and $\rho t_{m} \pi a^{2}$ is the total mass of the membrane. The term in square brackets is the square of rms velocity:

$$
v_{\mathrm{rms}}=\sqrt{\frac{1}{\pi a^{2}} \int_{0}^{2 \pi} \int_{0}^{a} v(r) v^{*}(r) r \mathrm{~d} r \mathrm{~d} \theta} .
$$

For a rigid piston $(n=0)$, both average and rms velocity are equal and the model parameters are the same. The relationship between the average velocity, $v_{\text {avg }}$, the $\mathrm{rms}$ velocity, $v_{\text {rms }}$, and the peak velocity at the center of the membrane, $v_{p}$, are

$$
v_{\mathrm{rms}}=\frac{n+1}{\sqrt{2 n+1}} v_{\mathrm{avg}} \quad \text { and } \quad v_{p}=(n+1) v_{\mathrm{avg}} .
$$

For $n=2,\left|v_{\mathrm{rms}}\right|^{2}=1.8\left|v_{\mathrm{avg}}\right|^{2}$ and by using (2)-(4),

$$
\begin{aligned}
E_{K} & =\frac{1}{2}\left(\rho t_{m} \pi a^{2}\right)\left|v_{\mathrm{rms}}\right|^{2} \\
& =\frac{1}{2}\left(\rho t_{m} \pi a^{2}\right) 1.8\left|v_{\mathrm{avg}}\right|^{2} .
\end{aligned}
$$

Hence, the kinetic energy of the membrane mass in (2) is preserved only if rms velocity, $v_{\text {rms }}$, is employed as the through variable. Otherwise, $L$, representing the mass in the model, must be 1.8 times the membrane mass when $v_{\text {avg }}$ is employed.

\section{RMS Equivalent Circuit Model Parameters}

To derive an equivalent circuit with the rms velocity as the through variable, we consider the mechanical impedance of a clamped membrane in vacuum. We begin by defining the mechanical impedance as the ratio of total power across the driven surface of the membrane and the square of $v_{\text {rms }}$,

$$
Z_{\mathrm{rms}}=\frac{P w_{\mathrm{Total}}}{\left|v_{\mathrm{rms}}\right|^{2}}=\frac{\int_{0}^{a} 2 \pi p(r) v^{*}(r) r \mathrm{~d} r}{\left|v_{\mathrm{rms}}\right|^{2}},
$$

where $p(r)$ is the normal force per unit area distribution on the driven surface. Assuming that $p(r)$ is constant across the membrane surface and $n=2$ for clamped membrane, this impedance expression becomes $\left|v_{\text {avg }}\right|^{2} /\left|v_{\text {rms }}\right|^{2}$ times the Mason's mechanical impedance obtained from the total force to average velocity ratio. $Z_{\mathrm{rms}}$ can readily be calculated by FEM analysis. Matching the slope of the impedance of an equivalent series $L C$ section to (7) at the resonance frequency reveals that $L_{\mathrm{rms}}$ is exactly equal to the mass of the membrane, rather than 1.8 times the mass as in Mason's circuit.

$$
L_{\mathrm{rms}}=\rho t_{m} \pi a^{2}
$$

Hence, the lumped inductance in the rms circuit models the effect of mass directly. To preserve the resonance frequency in vacuum, the capacitance in Mason's circuit representing the compliance of the membrane must be multiplied with $\left|v_{\mathrm{rms}}\right|^{2} /\left|v_{\mathrm{avg}}\right|^{2}=1.8$,

$$
C_{\mathrm{rms}}=1.8 \frac{\left(1-\sigma^{2}\right) a^{2}}{16 \pi Y_{0} t_{m}^{3}},
$$

where $Y_{0}$ is the Young's modulus, $\sigma$ is the Poisson's ratio of the membrane material, and $t_{m}$ is the membrane thickness. A correction to this formula may be necessary for membranes with $t_{m} / a>0.1$ as explained in [5].

If $v_{\mathrm{rms}}$ is employed in the "normalized power" expression instead of $v_{\text {avg }}$, we obtain the normalized radiation impedance $Z_{\text {Rrms }}$, depicted in Fig. 2, which is $\left|v_{\text {avg }}\right|^{2} /\left|v_{\text {rms }}\right|^{2}$ times the "normalized power" in [17]. Calculation of $Z_{\text {Rrms }}$ can be found in the Appendix.

\section{Fundamental Equations of the CMUT}

Static and harmonic analyses in FEM can predict the DC deflection and the fundamental velocity profile, respec- 
tively. We studied the membrane behavior using dynamic transient FEM analysis and at 80 discrete radial positions along the membrane surface, we fitted a sum of sinusoids, which consists of the fundamental and its first 5 harmonics, to each of the time-domain velocity data obtained at these 80 locations along the radius. Then, the amplitude distribution of each harmonic along the radius is used to find the velocity profile at that frequency. The phase of each harmonic is observed to be almost constant across the radius. We fitted (1) to the obtained velocity profile of each harmonic component and we observed that $n$ in (1) is dependent on the bias voltage and varies between 1.95 and 2.2. As the bias voltage increases, $n$ also increases. Nevertheless, the membrane velocity profile can be modeled quite accurately as a clamped radiator $(n=2)$, up to more than 2 times the series resonance frequency.

It is possible to derive a nonlinear analytical electromechanical model for the CMUT. When the CMUT is driven by a voltage $V(t)=V_{\mathrm{DC}}+V_{\mathrm{ac}}(t)$, the electrostatic force acting on the small ring of area $2 \pi r \delta r$ can be calculated by taking the derivative of the stored energy in the clamped capacitance

$$
\delta F(r, t)=\frac{1}{2} V^{2}(t) \frac{\mathrm{d}[\delta C(x(r, t))]}{\mathrm{d} x},
$$

where $x(r, t)$ is the membrane displacement normal to the surface and the capacitance of the ring is

$$
\delta C(x(r, t))=\frac{\varepsilon_{0} 2 \pi r \delta r}{t_{g}-x(r, t)} .
$$

Total force on the driven surface of the membrane is found by integrating (10) as $\delta r \rightarrow 0$

$$
F_{\text {tot }}(t)=\varepsilon_{0} \pi V^{2}(t) \int_{0}^{a} \frac{r \mathrm{~d} r}{\left[t_{g}-x_{p}(t)\left(1-r^{2} / a^{2}\right)^{n}\right]^{2}},
$$

where $t_{g}$ is the gap height, $\varepsilon_{0}$ is the free space permittivity, and $x_{p}(t)=x(0, t)$ is the peak displacement at the center of the membrane. Eq. (12) can be evaluated for $n=2$ as follows

$$
F_{\mathrm{tot}}(t)=\frac{C_{0} V^{2}(t)}{4 t_{g}}\left[\frac{t_{g}}{t_{g}-x_{p}(t)}+\frac{\tanh ^{-1}\left(\sqrt{\frac{x_{p}(t)}{t_{g}}}\right)}{\sqrt{\frac{x_{p}(t)}{t_{g}}}}\right],
$$

where $C_{0}=\varepsilon_{0} \pi a^{2} / t_{g}$. Note that when $x_{p}(t)<0$,

$$
\frac{\tanh ^{-1}\left(\sqrt{x_{p}(t) / t_{g}}\right)}{\sqrt{x_{p}(t) / t_{g}}}=\frac{\tan ^{-1}\left(\sqrt{-x_{p}(t) / t_{g}}\right)}{\sqrt{-x_{p}(t) / t_{g}}},
$$

which is a more useful expression for a simulator.

For small displacements around the point $x_{p}(t)=0$, Taylor series expansion of (13) provides a simpler mathematical interpretation, where the leading terms are

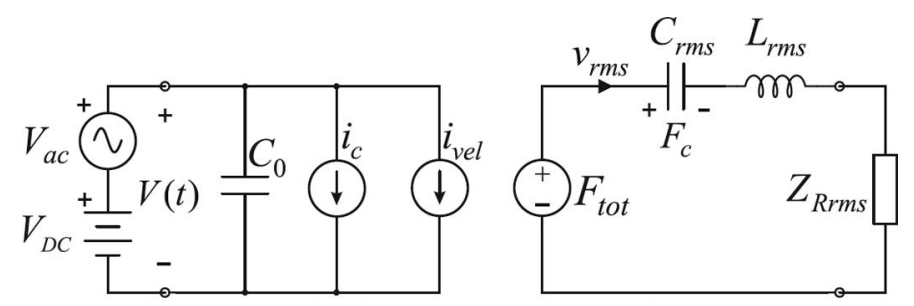

Fig. 3. Nonlinear large signal equivalent circuit. $i_{c}, i_{\text {vel }}$, and $F_{\text {tot }}$ are given by (18), (21), and (13) with $x_{p}(t)=\sqrt{5} C_{\mathrm{rms}} F_{c}(t) . L_{\mathrm{rms}}$ and $C_{\mathrm{rms}}$ are found by (8) and (9). $Z_{\text {Rrms }}$ is given in the Appendix.

$$
F_{\mathrm{tot}}(t) \approx \frac{C_{0} V^{2}(t)}{2 t_{g}}\left[1+\frac{2}{3} \frac{x_{p}(t)}{t_{g}}+\frac{3}{5}\left(\frac{x_{p}(t)}{t_{g}}\right)^{2}\right] .
$$

The current flowing through the electrodes of the small ring is the time derivative of the charge on this ring

$$
\frac{\mathrm{d}[\delta Q(r, t)]}{\mathrm{d} t}=\delta C(x(r, t)) \frac{\mathrm{d} V(t)}{\mathrm{d} t}+\frac{\mathrm{d}[\delta C(x(r, t))]}{\mathrm{d} t} V(t) .
$$

The first term in (16) is the capacitive current and the second one is induced by the membrane motion and, therefore, we call it the velocity current. We note that both current components depend on the instantaneous value of the membrane displacement. Considering an equivalent circuit, capacitive current flows through the shunt capacitance at the electrical side and velocity current is the one that gives rise to velocity at the mechanical port. To find the total capacitive current, $i_{\text {cap }}$, we evaluate the integral as $\delta r \rightarrow 0$

$$
\begin{aligned}
i_{\text {cap }}(t) & =\frac{\mathrm{d} V(t)}{\mathrm{d} t} \int_{0}^{a} \frac{2 \pi \varepsilon_{0} r \mathrm{~d} r}{t_{g}-x(r, t)} \\
& =C_{0} \frac{\mathrm{d} V(t)}{\mathrm{d} t} \frac{\tanh ^{-1}\left(\sqrt{x_{p}(t) / t_{g}}\right)}{\sqrt{x_{p}(t) / t_{g}}} .
\end{aligned}
$$

As shown in Fig. 3, this is the sum of currents in $C_{0}$ and a nonlinear component $i_{c}$. Hence, the nonlinear part is

$$
i_{c}(t)=C_{0} \frac{\mathrm{d} V(t)}{\mathrm{d} t}\left[\frac{\tanh ^{-1}\left(\sqrt{x_{p}(t) / t_{g}}\right)}{\sqrt{x_{p}(t) / t_{g}}}-1\right] .
$$

Taylor series expansion of $i_{c}$ gives

$$
i_{c}(t) \approx C_{0} \frac{\mathrm{d} V(t)}{\mathrm{d} t}\left[\frac{1}{3}\left(\frac{x_{p}(t)}{t_{g}}\right)+\frac{1}{5}\left(\frac{x_{p}(t)}{t_{g}}\right)^{2}\right] .
$$

To find the velocity current flowing through the clamped capacitance, the second term at the right hand side of (16) is rearranged and integrated over the membrane surface as $\delta r \rightarrow 0$

$$
i_{\text {vel }}(t)=2 \pi \varepsilon_{0} V(t) \frac{\mathrm{d} x_{p}(t)}{\mathrm{d} t} \int_{0}^{a} \frac{\left(1-r^{2} / a^{2}\right)^{2} r \mathrm{~d} r}{\left[t_{g}-x_{p}(t)\left(1-r^{2} / a^{2}\right)^{2}\right]^{2}},
$$


which is

$$
i_{\mathrm{vel}}(t)=\frac{C_{0} V(t)}{2 x_{p}(t)} \frac{\mathrm{d} x_{p}(t)}{\mathrm{d} t}\left[\frac{t_{g}}{t_{g}-x_{p}(t)}-\frac{\tanh ^{-1} \sqrt{\frac{x_{p}(t)}{t_{g}}}}{\sqrt{\frac{x_{p}(t)}{t_{g}}}}\right] .
$$

Taylor series expansion of the velocity current expression is

$$
i_{\mathrm{vel}}(t) \approx \frac{C_{0} V(t)}{t_{g}} \frac{\mathrm{d} x_{p}(t)}{\mathrm{d} t}\left[\frac{1}{3}+\frac{2}{5} \frac{x_{p}(t)}{t_{g}}+\frac{3}{7}\left(\frac{x_{p}(t)}{t_{g}}\right)^{2}\right]
$$

It is possible to obtain the small signal model parameters of the equivalent circuit from the Taylor series expansions of $F_{\text {tot }}, i_{c}$, and $i_{\text {vel. }}$. Assuming that the membrane displacement is very small compared with $t_{g}$ around $x_{p}=0$, we write from (15)

$$
F_{\text {tot }}(t) \approx \frac{V^{2}(t) C_{0}}{2 t_{g}}\left[1+\frac{2 x_{p}(t)}{3 t_{g}}\right]
$$

If we choose $V_{\mathrm{DC}} \gg V_{\mathrm{ac}}$, then $V^{2}(t) \approx V_{\mathrm{DC}}^{2}+2 V_{\mathrm{DC}} V_{\mathrm{ac}}(t)$, and because $x_{\text {avg }}(t)=x_{p}(t) / 3$, we find

$$
F_{\mathrm{tot}}(t) \approx \frac{V_{\mathrm{DC}}^{2} C_{0}}{2 t_{g}}+\frac{V_{\mathrm{DC}} C_{0}}{t_{g}} V_{\mathrm{ac}}(t)+\frac{V_{\mathrm{DC}}^{2} C_{0}}{t_{g}^{2}} x_{\mathrm{avg}}(t)
$$

where the first term at the right side represents the static force and the second term is the AC force due to electromechanical transformer ratio. The turns ratio of the transformer can be found from the second term as $N=V_{\mathrm{DC}} C_{0} /$ $t_{g}$ which is the same as that found in [1]. The third term in (24) is the amount of spring softening, due to increased electrostatic force caused by the displacement. It is like a negative capacitor of value $-C_{0} / N^{2}$, which is also consistent with [1].

For very small displacements around $x_{p}=0$, the nonlinear part, $i_{c}$, is negligible, and the velocity current is

$$
i_{\mathrm{vel}}(t) \approx \frac{C_{0} V_{\mathrm{DC}}}{3 t_{g}} \frac{\mathrm{d} x_{p}(t)}{\mathrm{d} t}=\frac{\sqrt{5} C_{0} V_{\mathrm{DC}}}{3 t_{g}} v_{\mathrm{rms}}(t) .
$$

The small signal parameters are sufficient to model a CMUT, as long as the membrane displacement is very small around $x_{p}=0$ and the spring softening is not very pronounced. However, CMUTs are always used with DC bias, and the assumption of operation around $x_{p}=0$ is not realistic even under small signal AC conditions. Also, it is apparent, even from the linearized equations, that the existence of large displacements can significantly alter the device behavior. To investigate the nonlinear nature of the CMUT, the unknown membrane displacement must be determined, so that the force and current equations can be implemented accordingly.

\section{Modeling of CMUT for Harmonic Balance ANALYSIS}

The harmonic balance (HB) analysis is a frequency domain nonlinear circuit analysis method, which is capable of finding the large signal, steady-state response of nonlinear circuits and systems. Linear circuits are modeled in frequency domain, while the nonlinear components are modeled with their time domain characteristics [20]. In this method, the input to the system is assumed to be a sinusoid, and the steady-state solution is found as the sum of a fundamental component and its harmonics. The method is significantly more efficient than time-domain simulators when the circuit contains components that are modeled in the frequency domain and the time constants are large compared with the period of the fundamental excitation frequency. In [21], a harmonic balance approach is applied to the weakly nonlinear equations of a MEMS microphone to characterize the unknown system parameters.

As seen in Fig. 3, we used a linear mechanical section and a consistent radiation impedance. A commercial harmonic balance simulator (Advanced Design System, Agilent Technologies, Palo Alto, CA) is used to implement the physical equations derived in Section III. We also performed transient simulations in addition to harmonic balance simulations and compared the results with FEM simulations.

The mechanical section is constructed as lumped elements and a component that encapsulates the radiation impedance, $Z_{\mathrm{rms}}(\omega)$, in the frequency domain with a suitable (Touchstone formatted) file. A symbolically defined device in the HB simulator enables us to create, multiport nonlinear equation-based components. We implemented the physical equations of the CMUT by relating port currents, port voltages, and their derivatives in this device. Eq. (13) is used to generate the total force, $F_{\text {tot }}$, at the mechanical side of the equivalent circuit. Eqs. (18) and (21) give $i_{c}$ and $i_{\mathrm{vel}}$ in the model of Fig. $3 ; x_{p}(t)$ in those equations represents the instantaneous charge in $C_{\mathrm{rms}}$, and it can be found from $x_{p}(t)=\sqrt{5} C_{\mathrm{rms}} F_{c}(t)$. When the absolute peak membrane displacement is $0.1 \%$ of the gap height or less, Taylor expansions of the equations are used to avoid convergence problems in the simulator that might occur when $x_{p}$ becomes very small. We can calculate total power, total force, and capacitive and velocity currents as outputs from the device.

\section{A. Static Analysis}

We compared the DC performance of the $\mathrm{HB}$ model with the FEM static analysis results. A CMUT membrane immersed in water with the top electrode placed at the bottom of the membrane is considered in all FEM simulations. Static deflection of a CMUT cell is examined with respect to DC bias voltage, the physical dimensions and the material properties of which are given in Table I. The CMUT collapses at $95 \mathrm{~V}$ in FEM analysis and at $97 \mathrm{~V}$ 
TABLE I. CMUt Dimensions and Constant Parameters Used in the Simulations.

\begin{tabular}{ll}
\hline Parameter & Value \\
\hline Radius, $a$ & $20 \mu \mathrm{m}$ \\
Gap height, $t_{g}$ & $0.25 \mu \mathrm{m}$ \\
Thickness of membrane, $t_{m}$ & $1 \mu \mathrm{m}$ \\
Collapse voltage, $V_{\text {col }}$ & $95 \mathrm{~V}$ \\
Poisson's ratio of $\mathrm{Si}_{3} \mathrm{~N}_{4}, \sigma$ & 0.263 \\
Density of membrane $\left(\mathrm{Si}_{3} \mathrm{~N}_{4}\right), \rho$ & $3.27 \mathrm{~g} / \mathrm{cm}^{3}$ \\
Young's modulus of $\mathrm{Si}_{3} \mathrm{~N}_{4}, Y_{0}$ & $3.2 \times 10^{5} \mathrm{MPa}$ \\
Density of water, $\rho_{0}$ & $1 \mathrm{~g} / \mathrm{cm}^{3}$ \\
Speed of sound in water, $c$ & $1500 \mathrm{~m} / \mathrm{sec}$ \\
\hline
\end{tabular}

in HB analysis, where $t_{m} / a=0.05$. In addition, increasing the thickness of the membrane, $t_{m}$, and repeating the analysis up to $t_{m} / a=0.2$ revealed that the amount of error is confined within $3 \%$. It must be noted that the choice of Mason's or rms mechanical section does not have any effect on the DC performance, and the membrane displacement is determined by the force model employed. It is noticed that the equivalent circuit predicts a higher peak displacement compared with FEM analysis.

The procedure is notably simplified while deriving the mechanical impedance of the CMUT with the assumption of constant force distribution in (7). The across variable is simply defined as total force on the membrane, $F_{\text {tot }}$, consistent with the phasor notation. However, there is an effect of nonuniform force distribution that becomes significant, particularly when the membrane is biased close to the collapse point. FEM analysis reveals that the force distribution is effectively uniform under low bias conditions, and a nonuniform component emerges as the bias is increased and becomes significant at high bias levels.

\section{B. Frequency Response Analysis}

1) Small-Signal Analysis: A prestressed harmonic analysis in FEM calculates the dynamic response of a biased membrane, assuming that the harmonically varying stresses are much smaller than the prestress itself. It does not take into account any kind of nonlinearity. Therefore, it is meaningful to evaluate only the small signal frequency response, where the membrane should not be biased very near to the collapse point. We can define the transducer's electrical admittance as $Y_{\text {in }}=G_{\text {in }}+j B_{\text {in }}$, where $G_{\text {in }}$ is the conductance and $B_{\mathrm{in}}$ is the susceptance of the CMUT. To follow the progress made in the proposed model, first, Mason's small signal equivalent circuit is analyzed. This circuit is composed of an electromechanical transformer with turns ratio, $N=V_{\mathrm{DC}} C_{0} / t_{g}$, which separates the spring softening capacitance, $-C_{0} / N^{2}$, and the mechanical $L C$ section defined in [4] from the shunt input capacitance, $C_{0}$. In Fig. 4(a), the small signal electrical conductance of an immersed CMUT cell is found by terminating this circuit with the radiation impedance of a piston, $Z_{\mathrm{Rp}}$. At bias voltages, $60 \mathrm{~V}, 70 \mathrm{~V}$, and $80 \mathrm{~V}$, a $1-\mathrm{V}$ peak AC signal is applied, and the results are compared with the FEM harmonic analysis results. At $V_{\mathrm{DC}}=90 \mathrm{~V}$, the membrane col-

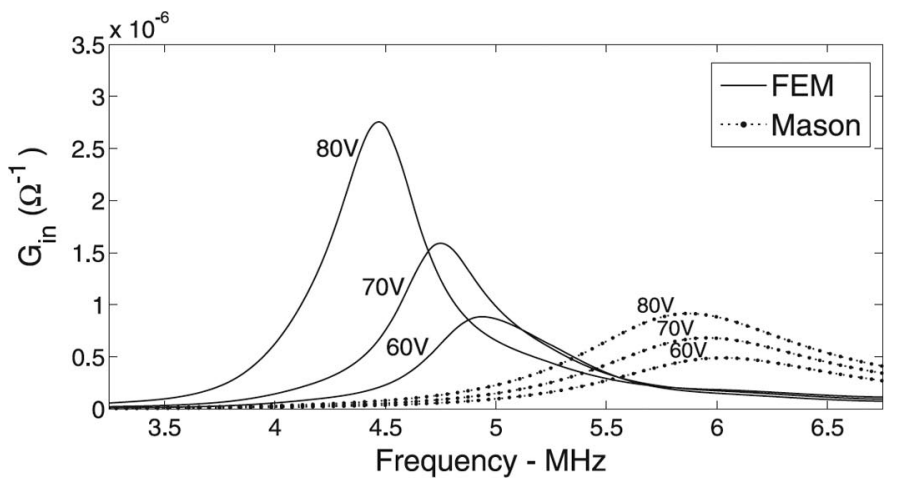

(a)

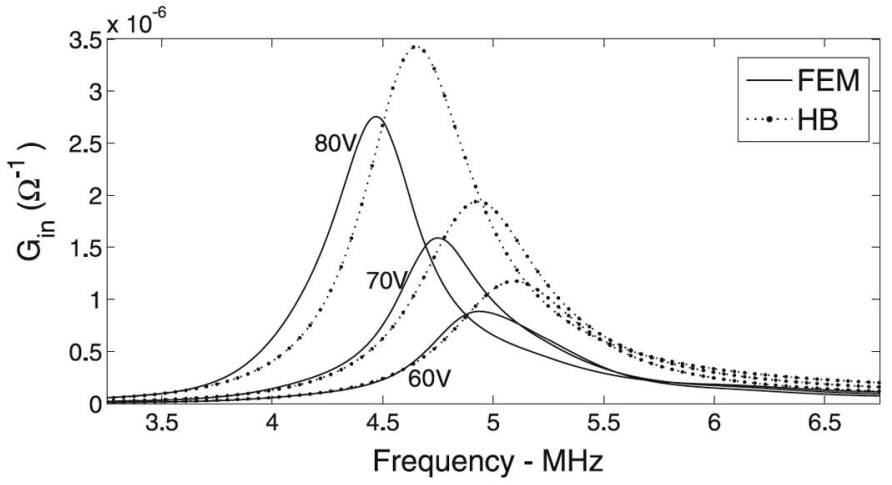

(b)

Fig. 4. Small signal electrical conductance of a CMUT cell in water; $1-\mathrm{V}$ peak AC signal is applied at bias voltages of $60 \mathrm{~V}, 70 \mathrm{~V}$, and $80 \mathrm{~V}$. Finite element method (FEM; solid) results are acquired from prestressed harmonic analysis and compared with (a) the frequency response of Mason's small signal equivalent circuit, which is terminated by the radiation impedance $Z_{\mathrm{Rp}}$ and (b) the harmonic balance (HB) analysis of the proposed equivalent circuit shown in Fig. 3.

lapses around the resonance frequency. It can be seen that the resonance frequencies are much lower and the spring softening is more pronounced in the FEM predictions.

Improvement is obtained when Mason's equivalent mechanical section and the corresponding radiation impedance is replaced by the rms equivalent mechanical section, $L_{\mathrm{rms}}$ and $C_{\mathrm{rms}}$, and the radiation impedance $Z_{\mathrm{Rrms}}$, respectively, as explained in Section II-C. This is the circuit shown in Fig. 3, where root mean square velocity, $v_{\text {rms }}$, and $F_{\text {tot }}$ are the through and across variables, respectively. The conductance obtained by this model for same bias levels is depicted in Fig. 4(b), together with the FEM results. Much better agreement is achieved in terms of peak conductance level, resonance frequency estimation, and the spring softening effect. However, in HB results, the amplitude of the conductance and the resonance frequency are slightly higher compared with FEM. For higher $V_{\mathrm{ac}} / V_{\mathrm{DC}}$, resonance frequency predictions of both analyses converge to each other. We have also observed similar results for CMUTs having the same radii but thicker membranes up to $t_{m} / a=0.2$. We can see that resonance frequency shift due to increased bias voltage falls short as the bias voltage increases, where the nonuniform force distribution begins to be significant and the clamped membrane velocity profile changes ( $n$ increases). These 2 major variations 


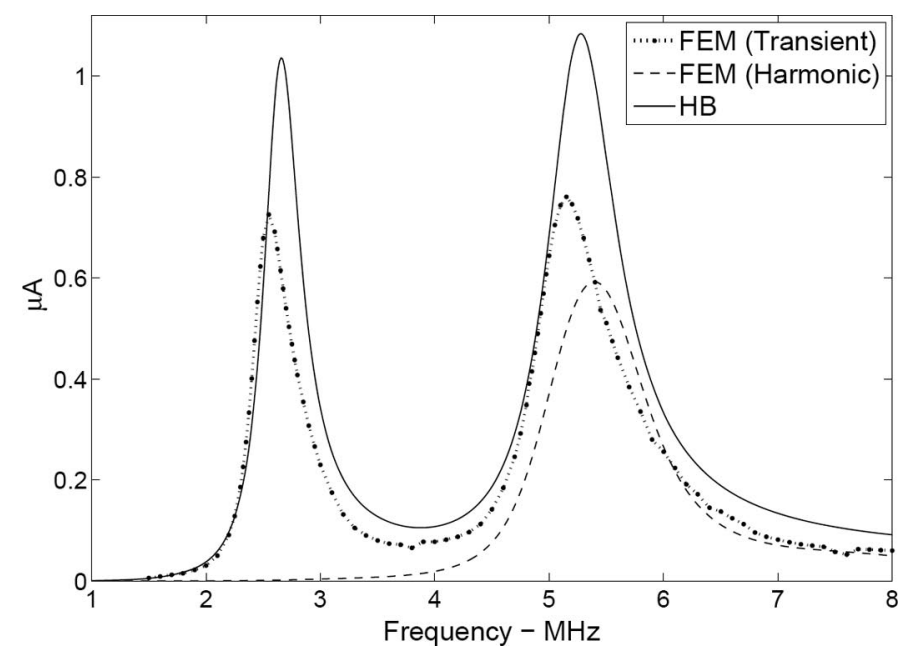

Fig. 5. Real part of the fundamental electrical source current of the CMUT cell in water for $V_{\mathrm{DC}}=10 \mathrm{~V}$ and a peak AC voltage of $40 \mathrm{~V}$. Large signal response is examined in finite element method (FEM), both with transient (dotted) and prestressed harmonic analysis (dashed). Root mean square equivalent circuit result is obtained from harmonic balance (HB; solid) simulation.

increase the error rate as we approach close to the collapse point.

We have also carried out the transient FEM analysis at several discrete frequencies to validate the linear behavior of the membrane under these drive conditions. We observed that the transient analysis yields exactly the same results with the prestressed harmonic analysis in FEM for linear operations.

2) Nonlinear Analysis: Prestressed harmonic analysis is reliable for small signal simulations. To find out the large signal performance of the introduced model, we employed dynamic transient analysis in FEM. On account of this, it is necessary to apply large $V_{\mathrm{ac}}$ as long as the membrane does not collapse during the transient process. In Fig. 5, the real part of the fundamental component of the source current is shown for HB analysis, together with transient and prestressed harmonic FEM analyses. In these simulations $V_{\mathrm{DC}}$ and $V_{\mathrm{ac}}$ are $10 \mathrm{~V}$ and $40 \mathrm{~V}$ peak, respectively. There is a peak at half the resonance frequency, because the second harmonic coincides with the resonance frequency causing significant membrane velocity at the resonance. However, prestressed FEM harmonic analysis cannot predict the nonlinear behavior. On the other hand, the rms equivalent circuit predicts the conduction peak at half the resonance frequency quite well. The data of the equivalent circuit for this figure is obtained in less than one minute, while producing the data of transient FEM analysis took approximately one day on the same computer.

\section{Transient Analysis}

In Fig. 6, the displacement at the center of the membrane is plotted, which is driven with a sinusoidal signal

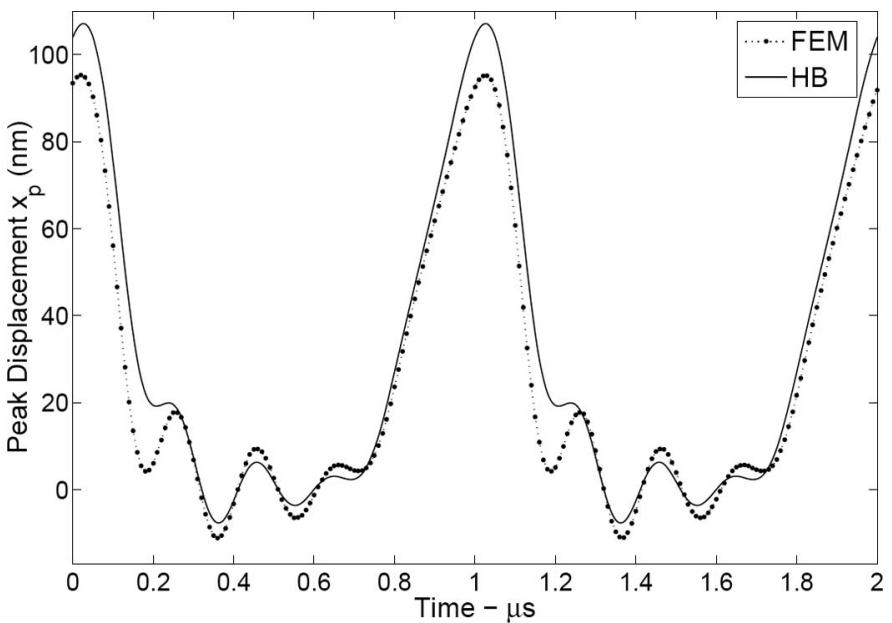

Fig. 6. Peak displacement of the CMUT cell in water, which is driven with a high sinusoidal voltage at a frequency one-fifth the resonance. Comparisons between transient analysis in finite element method (FEM; dotted) and harmonic balance (HB; solid) simulation of the nonlinear rms equivalent circuit are shown.

of $50-\mathrm{V}$ peak amplitude at $1 \mathrm{MHz}$, superimposed on $40-\mathrm{V}$ bias voltage. The drive frequency is approximately onefifth the resonance frequency of the CMUT cell. The HB solution converges within 1 second, which approximates the steady state of a transient FEM solution accurately. Nonlinear effects are very pronounced, because a large AC signal is employed.

Although the harmonic balance simulates the nonlinear circuits rapidly, it only produces the steady-state response. We also studied the transient effects of the same rms equivalent circuit by means of transient simulations. Fig. 7 shows the peak displacement of the membrane, where the membrane is biased to $40 \mathrm{~V}$ and a rectangular pulse of $40-\mathrm{V}$ amplitude is superimposed for $0.1 \mu \mathrm{s}$ duration. Although the agreement is impressive, FEM simulation predicts a slightly faster damping.

\section{Pulse Shaping}

Using the equivalent model equations and parameters, we can design the shape of the driving voltage to obtain the desired acoustic signal. A pressure pulse, which has a Gaussian-shaped frequency spectrum, is an appropriate choice at the surface of the membrane. First, the magnitude, the bandwidth, and the center of this spectrum are determined. Then, the rms velocity is found in the frequency domain, from the ratio of the desired total force at the top surface of the membrane and the impedance of the medium. The total force, $F_{\text {tot }}$, at the driven surface is calculated from the product of rms velocity and the total mechanical impedance. Finally, the driving voltage is calculated from (13).

If the desired pulse across the radiation impedance is as shown in Fig. 8(a), the model predicts the driving voltage as given in Fig. 8(b). To check the validity of this driving voltage, it is applied in FEM transient analysis, and the obtained result is also plotted in Fig. 8(a). The obtained 


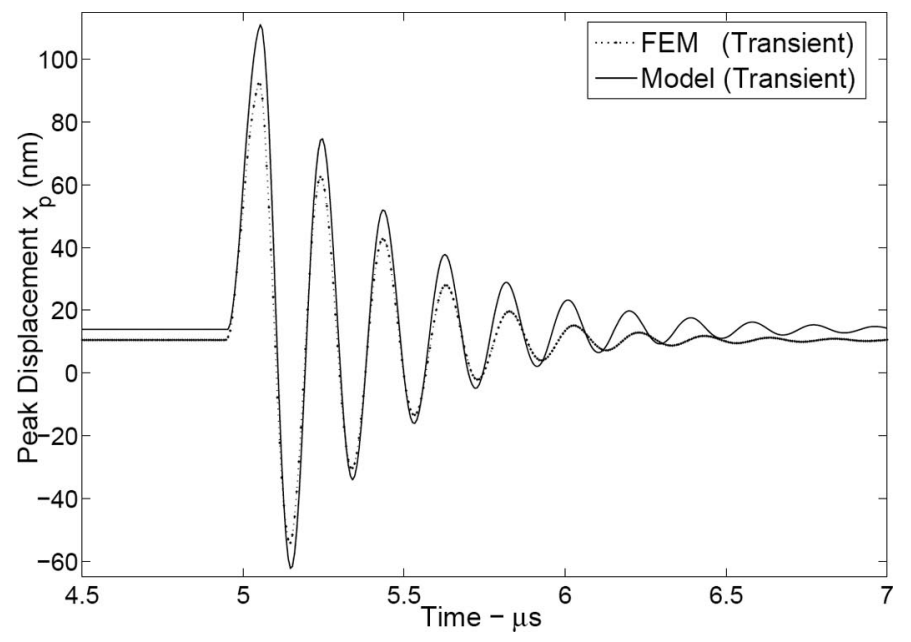

Fig. 7. Peak displacement of the CMUT cell in water, which is driven with a $0.1 \mu$ s pulse. $V_{\text {low }}=40 \mathrm{~V}, V_{\text {high }}=80 \mathrm{~V}$. Comparisons between the transient analyses carried out both in finite element method (FEM; dotted) and the nonlinear rms equivalent circuit (solid) are shown.

pulse shape is a little distorted compared with the expected one. However, the results are very similar both in FEM and HB. We note that calculating the driving voltage and obtaining the result in HB takes place in less than 1 minute. Although the frequency spectrum of the desired pulse is centered at $3 \mathrm{MHz}$ with a $6-\mathrm{dB}$ bandwidth of $1.2 \mathrm{MHz}$, the necessary drive voltage contains significant harmonics nearly up to $15 \mathrm{MHz}$. If a larger bandwidth is aimed centered at the resonance frequency, even more harmonics are needed at higher frequencies for the excitation voltage. The linear mechanical $L C$ section is valid over a wide frequency band around the resonance frequency, but it fails to represent the membrane dynamics as the frequency approaches to antiresonance. The antiresonance of this particular immersed membrane is around $25 \mathrm{MHz}$. As long as the frequency spectrum of the obtained driving voltage is confined in the valid operation band of the model, the obtained pulse shape will be very similar to the desired one.

\section{CONCLUSions}

An alternative equivalent circuit for immersed circular CMUT is presented for linear and nonlinear operations. Root mean square velocity is used instead of the commonly used average velocity. A clamped profile is used when deriving the analytical force and current equations, and the corresponding radiation impedance is employed. All parameters in the model can be simply calculated analytically for particular device dimensions, independent of FEM analysis. Although there is no parameter tuning in our model using the FEM results, the predictions are accurate.

Differences between FEM results and the model predictions can be accounted for the approximations in the

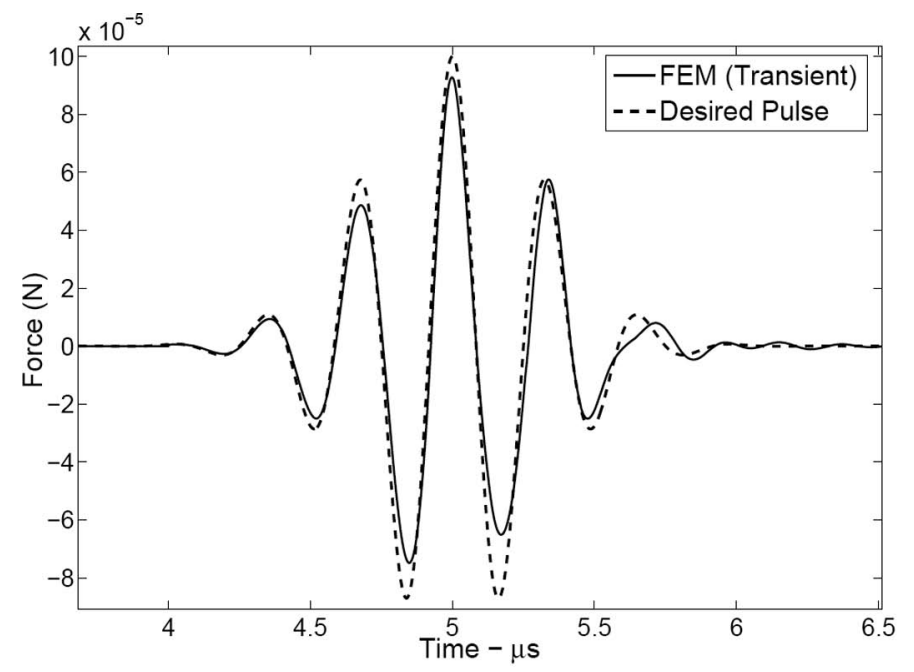

(a)

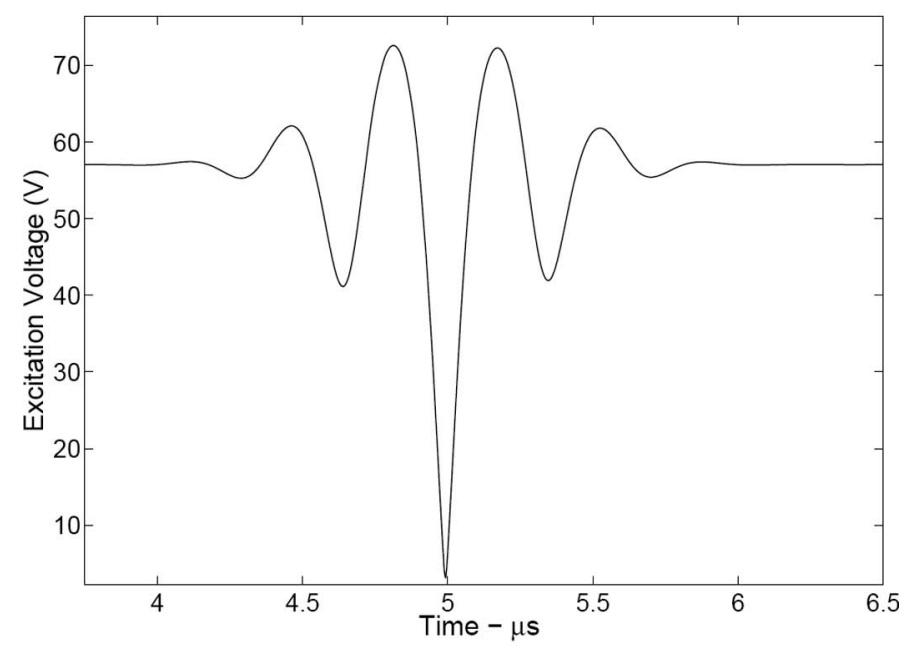

(b)

Fig. 8. (a) The desired pulse shape (dashed) and the achieved total force (solid) at the top surface of the CMUT in water, which is obtained when the designed voltage waveform in (b) is applied.

model equations. One of them is the variable nature of $n$ as a function of bias voltage. FEM analysis predicts that the clamped membrane deflection profile can be modeled by (1) for $n$ varying between 1.95 and 2.2. We have taken $n=2$. Furthermore, the force distribution across the membrane is not uniform. FEM simulations show that force is larger at the center of the membrane compared with its periphery. We used the total force on the membrane as the lumped across variable in the model, which estimates a lower deflection at the center.

\section{APPENDIX RADiATION IMPEDANCE}

Radiation impedance of a transducer with a certain velocity profile is the ratio of total power radiated from the 
acoustical terminals to the square of the absolute value of a nonzero reference velocity:

$$
Z_{R}=\frac{2 \pi \int_{0}^{a} P(r) v^{*}(r) r \mathrm{~d} r}{V_{i} V_{i}^{*}}=\frac{P_{\mathrm{TOTAL}}}{\left|V_{i}\right|^{2}}
$$

When rms velocity is chosen for $V_{i}$, the radiation impedance derived in [17] becomes

$$
Z_{\mathrm{Rrms}}=\pi a^{2} \rho_{0} c\left(1-\frac{20}{(k a)^{9}}\left[F_{1}(2 k a)+j F_{2}(2 k a)\right]\right),
$$

where $\rho_{0}$ is the density and $c$ is the speed of sound of the immersion medium and

$$
\begin{aligned}
F_{1}(y)= & \left(y^{4}-91 y^{2}+504\right) J_{1}(y)+14 y\left(y^{2}-18\right) J_{0}(y) \\
& -y^{5} / 16-y^{7} / 768,
\end{aligned}
$$

and

$$
\begin{aligned}
F_{2}(y)= & -\left(y^{4}-91 y^{2}+504\right) H_{1}(y)-14 y\left(y^{2}-18\right) H_{0}(y) \\
& +14 y^{4} / 15 \pi-168 y^{2} / \pi .
\end{aligned}
$$

$J_{n}$ and $H_{n}$ are the $n$th order Bessel and Struve functions, respectively.

For $k a<0.1, Z_{\mathrm{Rrms}}=R_{\mathrm{Rrms}}+j X_{\mathrm{Rrms}}$ can be approximated as

$$
Z_{\mathrm{Rrms}} \approx \frac{5}{9} \pi a^{2} \rho_{0} c\left[\frac{(k a)^{2}}{2}+j \frac{2^{16}}{17325 \pi}(k a)\right] .
$$

\section{REFERENCES}

[1] I. Ladabaum, X. Jin, H. Soh, A. Atalar, and B. Khuri-Yakub, "Surface micromachined capacitive ultrasonic transducers," IEEE Trans. Ultrason. Ferroelectr. Freq. Control, vol. 45, no. 3, pp. 678-690, May 1998.

[2] J. Knight, J. McLean, and F. Degertekin, "Low temperature fabrication of immersion capacitive micromachined ultrasonic transducers on silicon and dielectric substrates," IEEE Trans. Ultrason. Ferroelectr. Freq. Control, vol. 51, no. 10, pp. 1324-1333, Oct. 2004.

[3] Y. Huang, A. Ergun, E. Haeggstrom, M. Badi, and B. Khuri-Yakub, "Fabricating capacitive micromachined ultrasonic transducers with wafer-bonding technology," J. Microelectromech. Syst., vol. 12, no. 2, pp. 128-137, Apr. 2003.

[4] W. Mason, Electromechanical Transducers and Wave Filters, 2nd ed. New York: Van Nostrand, 1948.

[5] H. Koymen, M. Senlik, A. Atalar, and S. Olcum, "Parametric linear modeling of circular cMUT membranes in vacuum," IEEE Trans. Ultrason. Ferroelectr. Freq. Control, vol. 54, no. 6, pp. 1229-1239, Jun. 2007.
6] A. Lohfink and P.-C. Eccardt, "Linear and nonlinear equivalent circuit modeling of CMUTs," IEEE Trans. Ultrason. Ferroelectr. Freq. Control, vol. 52, no. 12, pp. 2163-2172, Dec. 2005.

[7] D. Certon, F. Teston, and F. Patat, "A finite difference model for cMUT devices," IEEE Trans. Ultrason. Ferroelectr. Freq. Control, vol. 52, no. 12, pp. 2199-2210, Dec. 2005.

[8] G. Yaralioglu, S. Ergun, and B. Khuri-Yakub, "Finite-element analysis of capacitive micromachined ultrasonic transducers." IEEE Trans. Ultrason. Ferroelectr. Freq. Control, vol. 52, no. 12, pp. 21852198, Dec. 2005.

[9] I. O. Wygant, M. Kupnik, and B. T. Khuri-Yakub, "Analytically calculating membrane displacement and the equivalent circuit model of a circular CMUT cell," in Proc. IEEE Ultrasonics Symp., Nov. 2008, pp. 2111-2114

10] S. Olcum, M. Senlik, and A. Atalar, "Optimization of the gainbandwidth product of capacitive micromachined ultrasonic transducers," IEEE Trans. Ultrason. Ferroelectr. Freq. Control, vol. 52 , no. 12 , pp. 2211-2219, Dec. 2005.

[11] M. Kupnik, I. O. Wygant, and B. T. Khuri-Yakub, "Finite element analysis of stress stiffening effects in CMUTs," in Proc. IEEE Ultrason. Symp., Nov. 2008, pp. 487-490.

[12] S. Wong, R. Watkins, M. Kupnik, K. Pauly, and B. Khuri-Yakub, "Feasibility of MR-temperature mapping of ultrasonic heating from a CMUT," IEEE Trans. Ultrason. Ferroelectr. Freq. Control, vol. 55, no. 4, pp. 811-818, Apr. 2008.

13] B. Bayram, G. Yaralioglu, M. Kupnik, A. Ergun, O. Oralkan, A. Nikoozadeh, and B. Khuri-Yakub, "Dynamic analysis of capacitive micromachined ultrasonic transducers," IEEE Trans. Ultrason. Ferroelectr. Freq. Control, vol. 52, no. 12, pp. 2270-2275, Dec. 2005

[14] J. Chen, X. Cheng, C.-C. Chen, P.-C. Li, J.-H. Liu, and Y.-T. Cheng, "A capacitive micromachined ultrasonic transducer array for minimally invasive medical diagnosis," J. Microelectromech. Syst. vol. 17, no. 3, pp. 599-610, Jun. 2008.

[15] I. Wygant, M. Kupnik, J. Windsor, W. Wright, M. Wochner G. Yaralioglu, M. Hamilton, and B. Khuri-Yakub, "50 khz capacitive micromachined ultrasonic transducers for generation of highly directional sound with parametric arrays," IEEE Trans. Ultrason. Ferroelectr. Freq. Control, vol. 56, no. 1, pp. 193-203 Jan. 2009.

[16] I. O. Wygant, M. Kupnik, B. T. Khuri-Yakub, M. S. Wochner, W M. Wright, and M. F. Hamilton, "The design and characterization of capacitive micromachined ultrasonic transducers (CMUTs) for generating high-intensity ultrasound for transmission of directional audio," in Proc. IEEE Ultrasonics Symp., Nov. 2008, pp. 21002102.

[17] M. Greenspan, "Piston radiator: Some extensions of the theory," $J$. Acoust. Soc. Am., vol. 65, no. 3, art. no. 608621, Mar. 1979.

[18] G. Yaralioglu, M. Badi, A. Ergun, and B. Khuri-Yakub, "Improved equivalent circuit and finite element method modeling of capacitive micromachined ultrasonic transducers," in Proc. IEEE Ultrasonics Symp., vol. 1, Oct. 2003, pp. 469-472.

[19] A. Bozkurt and M. Karaman, "A lumped circuit model for the radiation impedance of a 2D CMUT array element," in Proc. IEEE Ultrasonics Symp., vol. 4, Sep. 2005, pp. 1929-1932.

20] S. A. Maas, Nonlinear Microwave and RF Circuits, 2nd ed. Norwood, MA: Artech House, 2003

211] J. Liu, D. Martin, T. Nishida, L. Cattafesta, M. Sheplak, and B. Mann, "Harmonic balance nonlinear identification of a capacitive dualbackplate MEMS microphone," J. Microelectromech. Syst., vol. 17, no. 3, pp. 698-708, Jun. 2008.

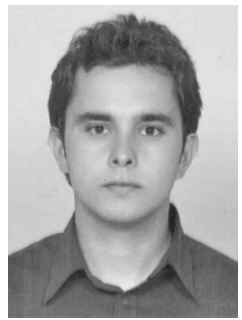

H. Kagan Oguz was born in Ankara, Turkey, in 1985. He received his B.S. and M.S. degrees in electrical engineering in 2006 and 2009, respectively, both from Bilkent University, Ankara, Turkey, where he was a research assistant.

He is currently working toward his Ph.D. degree in the same department and continues with Meteksan Defence Industry Inc. as a research engineer in underwater acoustic systems division. 


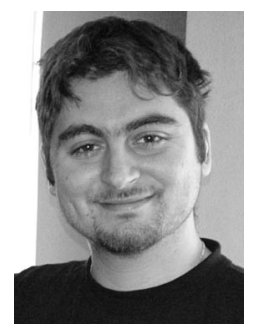

Selim Olcum was born in Chicago, IL, in 1981. He received his B.S. and M.S. degrees in electrical engineering in 2003 and 2005, respectively, both from Bilkent University, Ankara, Turkey.

He worked as a guest researcher at National Institute of Standards and Technology, Semiconductor Electronics Division during the summers of 2002 and 2003. He was a visiting scholar in the Micromachined Sensors and Transducers Laboratory of Georgia Institute of Technology, Atlanta, GA, in 2006. He is currently working toward his $\mathrm{Ph} . \mathrm{D}$. degree in electrical and electronics engineering at Bilkent University where he has been a research and teaching assistant since 2003.

His current research interests include optical and acoustical micromachined sensors and actuators. Mr. Olcum is a recipient of the ASELSAN Ph.D. Scholarship. He has been a member of IEEE and the UFFC Society since 2003 .

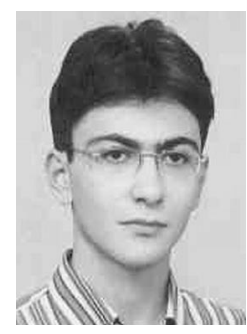

Muhammed N. Senlik was born in Isparta, Turkey, in 1981. He received his B.S. degree from Bilkent University, Ankara, Turkey, in 2002 in electrical engineering. He is currently working toward his Ph.D. degree in the same department, where he has been a research assistant since 2002.

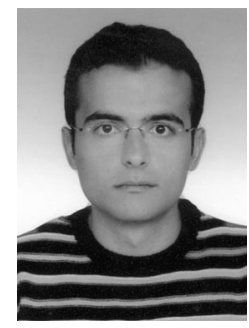

Vahdettin Tas received a B.S. degree from Middle East Technical University, Ankara, Turkey, in 2007 and an M.S. degree from Bilkent University, Ankara, Turkey, in 2009, both in electrical engineering.

He is currently working toward his Ph.D. degree in the Department of Electrical Engineering and Computer Science at the University of California, Berkeley, CA. His current research interests include micromechanical signal processors and atomic force microscopy.

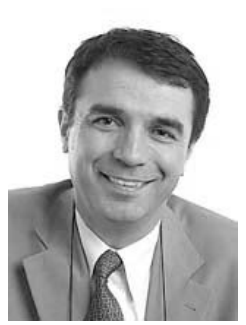

Abdullah Atalar received his B.S. degree from Middle East Technical University, Ankara, Turkey, in 1974, and his M.S. and Ph.D. degrees from Stanford University, Stanford, CA, in 1976 and 1978, respectively, all in electrical engineering. From 1978 to 1980 , he was first a postdoctoral fellow and later an engineering research associate at Stanford University. For 8 months he was with Hewlett Packard Labs, Palo Alto, CA. From 1980 to 1986 , he was on the faculty of the Middle East Technical University as an assistant professor. In 1986, he joined Bilkent University, Ankara, Turkey, as chairman of the Electrical and Electronics Engineering Department and served in the founding of the department, where he is currently a professor. He is presently the Provost of Bilkent University. During 1996-1998 he was a visiting professor at Stanford University. His current research interests include microwave electronics and micromachined sensors. He was awarded the Science Award of the Turkish Scientific Research Council (TUBITAK) in 1994. He is a Fellow of IEEE and a member of Turkish Academy of Sciences.

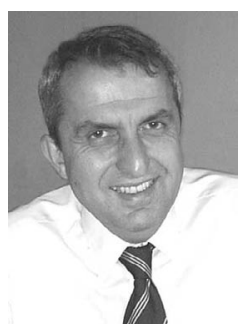

Hayrettin Köymen (M'87-SM'91) received the B.Sc. and M.Sc. degrees from Middle East Technical University (METU), Ankara, Turkey, in 1973 and 1976, respectively, and the Ph.D. degree from Birmingham University, Birmingham, UK, in 1979, all in electrical engineering.

He worked as a faculty member in the Marine Sciences Department (Mersin) and Electrical Engineering Department (Ankara) of METU from 1979 to 1990 . He has been a professor at Bilkent University, Ankara, Turkey, since 1990. His research activities have included underwater acoustic and ultrasonic transducer design, acoustic microscopy, ultrasonic NDT, biomedical instrumentation, mobile communications, and spectrum management.

Dr. Köymen is a fellow of IET (formerly IEE). 